\title{
Applicability problems for statistical methods in measuring problems of cosmology
}

Levin S.F.

Moscow institute of expertise and tests, Department of metrology and metrological support. Moscow, Russia; E-mail: Levin<AntoninaEL@ rostest.ru>;

The review of the problems arising because of incorrect formulation of measuring problems for identification cosmological models and infringement of applicability conditions for statistical methods of their decision.

Keywords: cosmology, statistical methods, infringements of applicability conditions.

DOI: $10.18698 / 2309-7604-2015-1-311-325$

\section{Introduction}

Problems of application for mathematical statistics in cosmology are coevals of Hubble law and, unfortunately, are still actual, though many of them have received mathematical, methodical and program decision during all-Union discussion of 1980-1990th years [1-8]. Foreign experts P. Huber, F. Mosteller, J. Tukey, I. Vuchkov, F. Hampel, etc. have taken part in discussion. Actually discussion was the answer to A.N. Kolmogorov's question on objective sense of probability. The subject of its discussion was catastrophic phenomenon of 1985-1986 in aviation, space-rocket and kern-power techniques its reasons [4]. It was unprecedented on mass character, synchronism of occurrence and suddenness a stream of refusals for difficult techniques. The phenomenon has caused confusion among experts. But then for first time among the reasons of accidents and failures have been found inadequacy errors of mathematical models, infringement of applicability conditions for statistical methods and out statement incorrectness of measuring problems. Then 24 standards on statistical methods from 31 and all standards on applied statistics have been disavowed.

Accuracy of astrophysical measurements for last decades has increased on usages, but the reasons of some cosmology problems there is an incorrectness of the formulation and infringement of applicability conditions for statistical methods at the decision of measuring problems of structurally-parametrical identification for mathematical models of physical objects. Most difficult of them is, naturally, astronomical Universe.

The known mathematical recipe [9] consists that «incorrect application of statistical methods can lead to the incorrect conclusions. All (it is possible, and not stated obviously) the assumptions concerning theoretical distribution, should be checked up. Debar is using the same 
sample for estimating and for verification. We will notice, at last, that statistical criteria cannot prove any hypothesis: they can specify only in«absence of refutation»»).

During discussion the prevention [9] about «absence of refutation» was specified by Eljasberg-Hampel paradox [10,11] according to which at any significance value the zero nonparametric hypotheses will be rejected, let even at very great volume of sample. The prevention rather «the same samples for estimation and for check» to equivalently following statement: «Distinctions in limiting same distributions the statistical at check of simple and difficult hypotheses are so essential what to neglect it is absolutely inadmissible» [12]. Therefore already by the end of XX century in mathematical statistics have refused statistical check of hypotheses at beforehand set significance value and have passed to «reached significance value» at multiplechoice check irrespective of used criterion of the consent.

The most known part of problems for applied statistics is connected with application of normal law with an average arithmetic the given measurements as «result of measurement» and «root-mean-square error (RMSE) of arithmetic mean» for statistical number of measurements. Problems of non-truncated probabilities distributions are less known. However the mess between tolerance and confidence intervals and «expanded uncertainty of measurement» and also between confidence probability, level of trust and probability of coverage became the most surprising on long duration. Eccentricity of mess underlines that the satisfactory definition of the tolerant interval which has appeared in [13], was absent in the international standard-prototype that it is impossible to tell about the formulas generating thereupon illusion of accuracy. On this background of scheme «cross examination» and «cross check» looked revolutionary, though from them to scheme of «cross observation for inadequacy error» [14] all one step.

The incorrectness of application of methods of statistics in measuring problems arises in method of indirect measurement - at use Taylor formula for nonlinear models, in method of cumulative measurements - at use of weight factors, normalized by dispersions of measured components, as «way of increase of accuracy» by association of rough results by scheme «non-uniformly» measurements contrary to scheme of maximum likelihood method, as dispersion average less than the least dispersion from components (non-identical incident), but itself average is an estimation only position parameter of mix of components distributions,

in method of collateral measurements - at application of regression analysis without check of performance of statistical uniformity conditions, gaussianity, non-correlated ness, non- 
confluence, i.e. by negligible casual errors of measurements for entrance variable mathematical models, and adequacy i.e. when the model structure is not neither superfluous, nor insufficient.

The most dangerous on consequences are infringements of those conditions of statistical methods applicability, physical and which mathematical sense is not clear to users. Those are infringements of stochastic compactness conditions for given measurements, similarity of the data presentation form to structure for accepted statistics and minimum of inadequacy error for mathematical models [14].

Stochastic compactness is generalization of statistical uniformity concept for the given repeated measurements of random variables on stochastic function.

It is known, that for an extended number of the given measurements presence of convergence of their selective distribution to general totality distribution is equivalent to condition of statistical uniformity for data. Its performance is promoted by repeated measurements on object in its same point, the same sizes, in the same conditions, the same copy of measuring apparatus, the same operator with identical carefulness during a short time interval.

Under an inadequacy error of mathematical models for physical objects long time believed an approximation error of the given measurements by model. Therefore requirements to it were normalized [15] earlier, than in [14] procedure of its identification within the limits of the cross observation scheme has been standardized.

Similarity between data presentation form and structure of statistics for nonparametric hypotheses check is the fullest is reached at representation of some measurements of random variable by statistical distribution function which is natural analogue for probabilities distribution function.

As a result infringement of applicability conditions of statistical methods, irrespective of measurements area, leads doubtful in the quantitative and qualitative relation to results. These circumstances in cosmology demand special consideration.

\section{Statistical problems in cosmology}

One of the first application problems of statistical methods in cosmology is the statistical heterogeneity problem for Hubble diagram.

On it characteristics of position for extragalactic objects of various morphological types (galaxies, radio galaxies and quasars) have standard value of inclination parameter $\theta_{1}=0,2$ at essential statistical disorder of red shift and the various zero-points connected with absolute magnitude of objects and Hubble parameter $H_{0}$. 
The problem was that removal from quasars sample of one object changed estimations of inclination parameter [16]. For sample [17] with MQM-estimation (Minimum Quadratic Method) of inclination parameter $\left.\theta_{1}\right|_{N=169}=0,100$ removal of allocated quasars gave $\left.\theta_{1}\right|_{N=168}=0,117$ and $\left.\theta_{1}\right|_{N=167}==0,177$. For quasars with spectra without features and reliably certain angular sizes the effect was even stronger: $\left.\theta_{1}\right|_{N=63}=0,1813 ;\left.\theta_{1}\right|_{N=62}=0,2380 ;\left.\theta_{1}\right|_{N=61}=0,2784$ [18].

The problem of applicability for statistical methods in cosmology is illustrated by «unexpected» results of data processing for astrophysical measurements:

- century drift of key parameter for cosmological models, Hubble's parameter $H_{0}=$ $530 \rightarrow 67 \mathrm{~km} \cdot \mathrm{s}^{-1} \cdot \mathrm{Mpc}^{-1}$ and acceleration parameter $q_{0}=+2,6 \pm 0,8 \rightarrow-1,0 \pm 0,4[17,19]$;

- global Euclidian geometry of the astronomical Universe [20];

- absence expected in Gaussian conditions the progress of accuracy for parameter estimations of $\Lambda \mathrm{CDM}$-model is proportional to a root square of volume of the given measurements;

- decrease in accuracy of indirect estimations for $H_{0}$ of within the limits of $\Lambda \mathrm{CDM}$-model from 1,28 to 6 times at the declared increase of accuracy of its adjustment to data of measurements at the expense of increase in number of parameter accordingly from $50 \%$ to $300 \%$ [21];

- dependence of own red shift of objects on their luminosity [22];

- coincidence dipole anisotropies of red shift, spatial heterogeneity of extragalactic sources and Galaxy polar axis [16, 23-25].

Dipole anisotropy of red shift in extragalactic sources spectra as first approximation is an example only statistical heterogeneity of the given astrophysical measurements. The second approach is connected with stochastic compactness of cosmological models. If in first case the account of angular co-ordinates of sources has allowed to establish, that continuation large-scale dipole anisotropies of red shift is the red-violet dipole of anisotropy in Local Super Congestion in the second case the account of own red shift of objects has led isotropy to cosmological component of red shift [26].

Stochastic compactness of objects models in method of collateral measurements is connected with applicability conditions for regression analysis [27, 14].

Them concern:

- stochastic compactness and non-confluence given measurements,

- limitlessness and isolation of systems of the equations of identification of model,

- non-correlated ness estimations of parameters of models, 
- centrality, homoscedastic and gaussianity of approximation errors of models, and the structure of models should not be superfluous or insufficient, i.e. inadequacy errors should be neglible small.

The most negative consequence of infringement of these conditions is stochastic multicollinearity [28] because of incorrect parameterization of variables and because absence of an optimality by criterion of minimum for inadequacy error of model. These consequences for standard cosmological $\Lambda$ CDM-models have received the name of «degeneration» [29].

The incident such, «degeneration» [30], has occurred to 6-parametrical standard cosmological $\Lambda$ CDM-model and results of statistical data processing of experiment WMAP at increase in number of parameters [31].

In $\Lambda$ CDM-model parameters are baryons density $\Omega_{b} \cdot h^{2}$, density of «cold dark matter» (CDM) $\Omega_{c} \cdot h^{2}$, density of «dark energy» $\Omega_{\Lambda}$ with condition index $\mathrm{w}=-1$, spectral index $n_{s}$, optical thickness to sphere of last dispersion $\tau$, and amplitude of fluctuations of galaxies density in radius $8 \mathrm{Mpc} \sigma_{8}$. Thus in parameters definition of baryons density and CDM Hubble's normalized constant $h$ or $H_{0} /\left(100 \mathrm{~km} \cdot \mathrm{s}^{-1} \cdot \mathrm{Mpc}^{-1}\right)$ is used. Command WMAP considered a parity of roots square of determinants of correlation matrixes for estimations of parameters according to measurements for 5 (WMAP-5) and 7 (WMAP-7) years. Then the conclusion has been drawn on increase of accuracy of identification of $\Lambda \mathrm{CDM}$-model by data for 7 years in comparison with data for 5 years in 1,5 times at 6 parameters, in 1,5 . 1,9 times at 7 parameters and in 3 times at 8 parameters (Table 1).

Table 1. Dependence of accuracy indirect estimation of Hubble's constant from number $Q \Lambda C D M-m o d e l$ parameters [30]

\begin{tabular}{|c|c|c|c|c|}
\hline \multicolumn{2}{|r|}{$\Lambda \mathrm{CDM}$-model with parameters $\Theta+$ additional parameters } & \multirow{2}{*}{$\begin{array}{c}Q \\
6\end{array}$} & \multirow{2}{*}{\begin{tabular}{c|}
$\begin{array}{c}\text { WMAP-5 } \\
\text { WMAP-7 }\end{array}$ \\
1,5
\end{tabular}} & \multirow{2}{*}{$\begin{array}{c}h \\
0,710 \pm 0,025\end{array}$} \\
\hline$\overline{\mathrm{ACDM}}$ & $\left\langle\Omega_{b} \cdot h^{2}, \Omega_{c} \cdot h^{2}, \Omega_{\Lambda}, \sigma_{8}, n_{\mathrm{s}}, \tau\right\}=\Theta$ & & & \\
\hline$\Lambda \mathrm{CDM}+r$ & $\Theta+$ tensor-scalar relation & 7 & 1,9 & $0,675 \pm 0,038$ \\
\hline$\Lambda \mathrm{CDM}+\frac{d n_{s}}{d \ln k}$ & $\Theta+$ logarithmic derivative of spectral index & 7 & 1,7 & $0,735 \pm 0,032$ \\
\hline$\Lambda \mathrm{CDM}+r+\frac{d n_{s}}{d \ln k}$ & $\begin{array}{l}\Theta+\text { tensor-scalar relation }+ \text { logarithmic derivative of spectral } \\
\text { index }\end{array}$ & 8 & 3,0 & $0,691^{+0,040} /-0,041$ \\
\hline$\Lambda \mathrm{CDM}+\alpha_{-1}$ & $\Theta+$ anti-correlated isocurvature modes CDM & 7 & 1,9 & $0,745^{+0,031 /-0,030}$ \\
\hline$\Lambda \mathrm{CDM}+\alpha_{0}$ & $\Theta+$ uncorrelated isocurvature modes CDM & 7 & 1,9 & $0,736 \pm 0,032$ \\
\hline$\Lambda \mathrm{CDM}+\mathrm{N}_{\mathrm{eff}}$ & $\Theta+$ neutrino mass & 7 & 1,8 & $0,826^{+0,089} /-0,087$ \\
\hline$\Lambda \mathrm{CDM}+\Omega_{k}$ & $\Theta+$ spatial curvature & 7 & 1,8 & $0,53^{+0,13 /-0,15}$ \\
\hline$\Lambda \mathrm{CDM}+w$ & $\Theta+$ dark energy equation of state & 7 & 1,5 & $0,75^{+0,15 /-0,14}$ \\
\hline
\end{tabular}


At the same time as it is good, as well as the standard cosmological $\Lambda$ CDM-model, to data WMAP satisfies cosmological model with quintessence at the condition equation $\mathrm{w}=-0,5$ with parameters $\Omega_{\mathrm{M}}=0,47$ and $H_{0}=57 \mathrm{~km} \cdot \mathrm{s}^{-1} \cdot \mathrm{Mpc}^{-1}$. But also it has been rejected, since the value of a constant of Hubble received in its frameworks on two mean square deviation less than its value in Hubble Space Telescope Key Project [32]; as the model with nonplanar space has been rejected also at $H_{0}=32,5 \mathrm{~km} \cdot \mathrm{s}^{-1} \cdot \mathrm{Mpc}^{-1}, \Omega_{\Lambda}=0$ and $\Omega_{\text {total }}=1,28$ [33].

In other words, an essential indicator of accuracy cosmological models actually is accuracy of indirect estimation for Hubble's constant.

Last column of Table 1 describe accuracy of $\Lambda \mathrm{CDM}$-model in the presence of additional parameters, shows, that accuracy indirect estimation within the limits of model of a constant of Hubble at increase in number of parameters has decreased in 1,28...6 times.

And in report WMAP for 9 years of measurements [34] this incident was not mentioned any more. Moreover, expected in conditions gaussianity specifications of estimations for parameters of $\Lambda \mathrm{CDM}$-model, inversely proportional to a root square of volume of data, have not occurred [35], some progress has been reached according to mission Plank (Table 2).

Table 2. Estimations of constant of Hubble $H_{0}, \mathrm{~km} \cdot \mathrm{c}^{-1} \cdot \mathrm{Mpc}^{-1}[34,36,37]$

\begin{tabular}{|c|c|c|c|c|c|c|}
\hline WMAP-1 & WMAP-3 & WMAP-5 & WMAP-7 & WMAP-9 & Plank 21.03.2013 & Plank 12.12.2013 \\
\hline $72 \pm 5$ & $73,2^{+3,1} /_{-3,2}$ & $71,9^{+2,6} /_{-2,7}$ & $71,0 \pm 2,5$ & $70,0 \pm 2,2$ & $67,9 \pm 1,5$ & $67,3 \pm 1,2$ \\
\hline
\end{tabular}

In the consent with the mathematical recipe [9] logicians of statistical conclusion in method of collateral measurements [14] as the zero consider hypotheses of degeneracy Ho (absence of dependence), continuity $\mathbf{H}_{00}$ (absence of changes of structure and parameters - «disorders») and composite uniformity Ho00 (existence of uniform model of the given measurements from various sources). Using of combination for method of repeated measurements and method of collateral measurements at identification of mathematical models of measurements objects and check of the listed hypotheses is based on continuity and stochastic compactness of models. Criterion of preference is the minimum of the average module of inadequacy error $\bar{\varepsilon}$ (AMIE) as average absolute deviation (AAD) $d$ the given measurements from the position characteristic of model in the cross observation scheme. This scheme allows reducing essentially restrictions on conditions of applicability for regression analysis algorithms. Identification algorithms used on the basis of this scheme name cross, and in aggregate with the described logic of statistical conclusion method of compactness maximum (MCM) [14]. 
For competing models with equal number of parameters is admissible to use AAD approximation errors though it is obvious, that in most cases $\bar{\varepsilon}>d$.

To the described logic of statistical conclusion the MQM-algorithms (MCMMQM), the AAD-algorithms (MCMAAD) and the algorithms of cross sliding median (MCMMEDS) [14] are subordinated. These algorithms provide consecutive complication of models by increase in number of parameters at consecutive search of binary codes of the structure which categories are tracer functions of corresponding parameters of model $\theta_{j} \neq 0$. Such procedure as a result allocates a structure model code of optimum complexity by criterion of minimum AMIE.

\section{Problem of calibration of a scale of distances}

In frameworks of a statistical conclusion logic [14] we will consider a problem of parametrical identification (calibration) on modules of photometric distance $\mu_{p}=5 \cdot \lg D_{L}+25 D_{L}$ supernovae SN Ia cosmological distances scales on red shift $z$ in model Friedman-RobertsonWalker [38]:

$$
\begin{aligned}
& D_{Z}=\frac{c \cdot(1+z)}{H_{0} \cdot\left|\Omega_{k}\right|^{1 / 2}} \cdot \operatorname{Six}\left\{\left|\Omega_{k}\right|^{1 / 2} \int_{0}^{z}\left[(1+z)^{2}\left(1+\Omega_{M} \cdot z\right)-z(2+z) \cdot \Omega_{\Lambda}\right]^{-1 / 2} d z\right\} \\
& \operatorname{Six}\{\cdot\}=\left\{\begin{array}{l}
\operatorname{sh}\{\cdot\}, \Omega_{k} \geq 0 \\
\sin \{\cdot\}, \Omega_{k} \leq 0
\end{array}\right.
\end{aligned}
$$

where $\Omega_{k}=1-\Omega_{M}-\Omega_{\Lambda}, \Omega_{M}$ - density of weights, $\Omega_{\Lambda}$ - density « $\Lambda$-energy». For 37 supernovae SN Ia model (1) is characterized AAD of approximation errors $d_{\Omega}=0,1456$.

Let's present now data [39] about distance modules of SN Ia samples from 27 supernovae SN Ia at $\lg c z=3,398 \ldots 4,572$ and 10 supernovae SN Ia at $\lg c z=4,954 \ldots 5,464$, but for the description of Hubble diagram it is used logarithmic model on the basis of radial velocity for objects:

$$
\mu_{p}(z)=\theta_{0}+\sum_{j=1}^{J} \theta_{j} \cdot(\lg c z)^{j}
$$


Check of hypothesis $\mathbf{H}_{0}$ and alternative hypotheses for model (2) has shown, that more plausible in comparison with MCMMEDS-estimation is MCMMQM-estimation at AAD $d_{\mu}=$ $0,1449^{m} \approx d_{\Omega}$ (Table 1$)$.

Table 1. Check of hypothesis $\mathrm{H}_{0}$ for the module of distance SN Ia [39] in a class of models (2) $J$ $=7$

\begin{tabular}{|c|c|c|c|c|c|c|c|c|c|c|}
\hline \multirow{2}{*}{ Algorithm } & \multirow{2}{*}{$\begin{array}{c}\text { Model } \\
\text { code }\end{array}$} & \multicolumn{7}{|c|}{ Parameters of continuous models } & AMIE \\
\cline { 3 - 10 } & & $\theta_{0}$ & $\theta_{1}$ & $\theta_{2}$ & $\theta_{3}$ & $\theta_{4}$ & $\theta_{5}$ & $\theta_{6}$ & $\theta_{7}$ & \\
\hline & 1111110 & 15,9878 & 3,62405 & 0,72754 & - & - & $4,186152 \cdot 10$ & 0 & 0 & 0,176768 \\
MCMMQM & 0 & 5 & 5 & 7 & 0,0458941 & $2,921237 \cdot 10$ & -3 & & 2 \\
& & & & & & -2 & & & \\
\hline MCMMED & 1010000 & 26,3792 & 0 & 0,59914 & 0 & 0 & 0 & 0 & 0,208567 \\
S & 0 & 8 & & 8 & & & & & 6 \\
\hline
\end{tabular}

Identification with the account «disorder» and composite heterogeneity shows (Table 2), that the method choice оценивания model parameters essentially influences result of structurallyparametrical identification: the MCMMQM-estimation shows significant statistical heterogeneity of data [39] that will be co-coordinated with a conclusion [40]. At the same time the MCMMEDSestimation specifies in statistical uniformity of data about photometric modules of distance SN Ia at «small» and «big» red shift.

The analysis of the MCMMEDS-estimation with same number of free parameters, as well as at model (1), by means of program «MRM-check 2.0» [41] has shown, that from among the truncated typical distributions by the most plausible it has appeared not Gauss distribution used in [39], and truncated Laplace distribution. For it within the limits of maximum likelihood method in parameter of dispersion the standard deviation and its estimation not in the form of RMSE, and an AAD.

And after all on factors of likelihood function for (1) in [39] the «non-identical incident», generating natural question was obvious: the received estimation $\sigma$ is that? RMSE, RMSE an average arithmetic or RMSE average weighted on dispersions?

In other words, the conclusion about «acceleration of Universe expansion» demands an additional substantiation, the analysis of an inadequacy error of model (1) and specification of a kind of probabilities distribution of deviations from it used data.

At the same time questions on observance of conditions of applicability of statistical methods and sense of the results received in frameworks of «the normal theory» by «the best fit $\chi^{2} \gg$ on the basis of Fisher's matrix, RMSE an average arithmetic and RMSE average weighted on 
dispersions, in [39] remained without the answer. There are questions on solvency of statistical criteria at infringement of preconditions «gaussianity» and about communication for distance modules of supernovae SN Ia with «horizon of events».

Table 2. Check of hypotheses $\mathrm{H}_{00}$ and $\mathrm{H}_{000}$ for dependence of the module of distance SN Ia according to [39] in class polynomial logarithmic models

\begin{tabular}{|c|c|c|c|c|c|c|c|c|}
\hline Algorithm & \multicolumn{8}{|c|}{ MCMMQM } \\
\hline $\begin{array}{c}\text { Range of } \\
\text { stochastic } \\
\text { compactness }\end{array}$ & \multicolumn{7}{|c|}{$3,398-4,572$} & $4,954-5,464$ \\
\hline $\begin{array}{c}\text { Continuity } \\
\text { interval }\end{array}$ & $3,398-3,685$ & $3,734-3,859$ & $3,871-3,891$ & \multicolumn{2}{|c|}{$3,896-4,178$} & \multicolumn{2}{|c|}{$4,189-4,572$} & $4,954-5,464$ \\
\hline Sample volume & 5 & 4 & 2 & \multicolumn{2}{|r|}{9} & \multicolumn{2}{|c|}{7} & 10 \\
\hline Model code & 10000000100 & 10000000001 & 01000000000 & \multicolumn{2}{|c|}{10000010000} & \multicolumn{2}{|c|}{10000000001} & 10000001000 \\
\hline $\begin{array}{c}\text { Model } \\
\text { parameters }\end{array}$ & $\begin{array}{c}31,70304 \\
7,442311 \cdot 10^{-5}\end{array}$ & $\begin{array}{c}32,92661 \\
3,349388 \cdot 10^{-6}\end{array}$ & 9,091724 & \multicolumn{2}{|c|}{34,02461} & 35,75944 & & $\begin{array}{c}38,18179 \\
4,265624 \cdot 10^{-5}\end{array}$ \\
\hline AMIE & $4,606171 \cdot 10^{-2}$ & $3,076077 \cdot 10^{-2}$ & $1,183701 \cdot 10^{-2}$ & \multicolumn{2}{|c|}{0,1385603} & \multicolumn{2}{|c|}{0,1357891} & 0,1408153 \\
\hline General AMIE & \multicolumn{8}{|c|}{$\mathbf{0 , 1 0 7 6 4 1 8 7 9 7}$} \\
\hline \multicolumn{3}{|c|}{ Algorithm } & \multicolumn{6}{|c|}{ MCMMEDS } \\
\hline \multicolumn{3}{|c|}{ Range of stochastic compactness } & \multicolumn{6}{|c|}{$3,398-5,464$} \\
\hline \multicolumn{3}{|c|}{ Continuity interval } & \multicolumn{2}{|c|}{$3,398-3,625$} & \multicolumn{2}{|c|}{$3,679-3,734$} & & $3,779-5,464$ \\
\hline \multicolumn{3}{|c|}{ Sample volume } & \multicolumn{2}{|l|}{3} & \multicolumn{2}{|c|}{3} & & $21+10$ \\
\hline \multicolumn{3}{|c|}{ Model code } & \multicolumn{2}{|c|}{10100000000} & \multicolumn{2}{|c|}{01000000000} & & 11000000001 \\
\hline \multicolumn{3}{|c|}{ Model parameters } & $\begin{array}{c}26,41511 \\
0,5710946\end{array}$ & & \multicolumn{2}{|c|}{9,294743} & & $\begin{array}{c}16,90781 \\
4,746854 \\
7,149604 \cdot 10^{-8}\end{array}$ \\
\hline \multicolumn{3}{|c|}{ AMIE } & \multicolumn{2}{|c|}{$1,21816 \cdot 10^{-3}$} & \multicolumn{2}{|c|}{$3,035736 \cdot 10^{-2}$} & & 0,1555352 \\
\hline \multicolumn{3}{|c|}{ General AMIE } & \multicolumn{6}{|c|}{0,132873453} \\
\hline
\end{tabular}

Unfortunately, «Fisher's statistical recipe» under the specification of parametrical models by Student's and Fisher's criterions for hypothesis «gaussianity» conducts to Eljasberg-Hampel paradox. And in this «recipe» are not present words about absence of independence and catastrophic non-robustness $F$-criterion (Fisher's criterion). Are not present words that various criteria of the consent estimate various and often insignificant aspects of fitting that at the big sample all level criteria exceed a significance value. And words that statistically «smooth» zero hypothesis to prove it is impossible, it can be denied only, though and at great volume of sample $[10,11]$. And even «following R. Fisher, with known care it is possible to tell, that an information matrix (Fisher's matrix - author insert) describes average quantity of the information on parameters 
of the distribution law, containing in casual sample» [42]. And after all in practice the law of deviations distribution of measurements from interpreting cosmological model, as rule, remains unknown.

Authors [39] have been puzzled by problems of statistics in 1996 because researchers considered themselves as beginners in this area. And though in A. Riss's dissertation has suggested to turn criterion $\chi^{2}$ into a certain probability with weight factors on dispersions of components [43], i.e. in function of credibility for so-called the «non-uniformly» measurements, already known problems for that moment «statistical $\chi^{2} »$ and hypotheses «normality» remained unmarked.

Besides, errors of inadequacy of model (1) were not estimated and to results of calibration on the same data for alternative models were not compared, that would represent not smaller interest, than only estimations of parameters $\Omega_{M}$ and $\Omega_{\Lambda}$. Therefore one more «unexpected» result has presented interpolation model with parameter of the form [18].

Use of this model for interpretation of data [38] has qualitatively confirmed presence of significant nonlinearity of dependence of red shift from photometric distance; however for data about the same supernovae of catalogue [44] interpretation model with parameter of the form of such result has not given.

\section{Conclusion}

In measuring problems of cosmology a number of infringements of conditions of statistical methods applicability takes place:

1) absence of statistical check of structural hypotheses,

2) indistinguishability simple and difficult hypotheses,

3 ) overestimate of probability of the consent by grouping of data for criterion $\chi^{2}$,

4) scheme application «non-uniformly precise» measurements and the square-law criteria sensitive to allocated results,

5) noncompliance with the terms of the statistical homogeneity for the sample data.

The formulation incorrectness of measurement problem for structural-parametric identification of cosmological models is associated with the selection accuracy criteria of the models without taking into account the inadequacy errors.

Each of these infringements even separately raises the doubts in estimations of structure and parameters of cosmological models, let alone conclusions. 


\section{Thanks}

To Braginsky Vladimir Borisovich - for support in the beginning of researches on subjects of application of statistical methods in cosmology.

To Vladimirov Jury Sergeevich - for useful discussion of problem at seminars «Physics and geometry».

To Ser Roger Penrose - for correctness and diplomacy answer to question on interpretation of «unexpected» results in cosmology for the «Big explosion» theory.

\section{References}

1. Alimov Ju.I. (1980). Al'ternativa metodu matematicheskoj statistiki [Alternative to mathematical statistics method]. Moscow: Knowledge.

2. Voprosy kibernetiki. VK-94 [Cybernetics questions. CQ-94]. (1982). Moscow: Academy of sciences USSR.

3. Levin S.F., Blinov A.P. (1988). Theoretical foundations of guaranteed error bounds for the solution of metrological problems by statistical methods. Measurement Techniques, Vol. 31, № 12, 1145-1150.

4. Levin S.F. (1989). Garantirovannost' programm obespechenija jekspluatacii tehniki [Guarantee support for program of techniques maintenance]. Metodicheskie rekomendacii [Methodical recommendations]. Kiev: Knowledge.

5. Statisticheskaja identifikacija, prognozirovanie i kontrol' [Statistical identification, prediction and verification]. (1990). Metodicheskie rekomendacii 1-go Vsesojuznogo seminara [Methodical recommendations of $1^{\text {st }}$ All-Union seminar]. Moscow: Ministry of Defense USSR.

6. Statisticheskaja identifikacija, prognozirovanie i kontrol' [Statistical identification, prediction and verification]. (1991). 2-j Vsesojuznyj nauchno-tehnicheskij seminar. Tezisy dokladov [Theses of reports for $2^{\text {nd }}$ All-Union seminar]. Sevastopol: Knowledge.

7. Levin S.F. (1991). Metrological certification and maintenance of software for statistical processing of results of measurements. Measurement Techniques, vol. 34, № 12, 1221-1225.

8. Levin S.F. (1995). Compactness maximum method and complex measurement problems. Measurement Techniques, vol. 38, № 7, 732-743.

9. Korn G.A., Korn T.M. (1961). Mathematical Handbook for Scientists and Engineers. New York, Toronto, London: W-Hill Book Company. 
Proceedings of International Conference PIRT-2015

10. Eljasberg P.E. (1983). Izmeritel'naja informacija: skol'ko ee nuzhno? kak ee obrabatyvat'? [The measuring information: how many it is necessary? How it to process?]. Moscow: Science.

11. Hampel F.R., Ronchetti E.M., Rousseeuw P.J., Stahel W.A. (1986). Robust Statistics: The Approach Based on Influence Functions. New York, Chichester, Brisbane, Toronto, Singapore: John Wiley \& Sons.

12. Prikladnaja statistika. Pravila proverki soglasija opytnogo raspredelenija s teoreticheskim [Applied statistics. Rules of check of experimental and theoretical distribution of the consent. Part II. Nonparametric goodness-of-fit test]. (2002).

13. Neopredelennost' izmerenija [Uncertainty of measurement]. Part 3: Guide to expression of uncertainty in measurement. (1995).

14. Gosudarstvennaja sistema obespechenija edinstva izmerenii. Opredelenie harakteristik matematicheskih modelej zavisimostej mezhdu fizicheskimi velichinami pri reshenii izmeritel'nyh zadach. Osnovnye polozhenij [State system for ensuring the uniformity of measurements. Determination of characteristics of mathematical models for dependences between physical values at the decision of measuring problems. Main principles]. (2000).

15. Gosudarstvennaja sistema obespechenija edinstva izmerenii. Izmerenija fizicheskih velichin. Osnovnye polozhenij [State system for ensuring the uniformity of measurements. Measurements of physical sizes. The general requirements]. (1980).

16. Burbidge G., Burbidge M. (1967). Quasi-stellar objects. S.-Francisco, London: Freeman \& Co.

17. Lang K.R. (1974). Astrophysical formulae. V.2. Berlin, Heidelberg, N.Y.: Springer-Verlag.

18. Levin S.F. (1980). Optimal'naja interpoljacionnaja fil'tracija statisticheskih harakteristik sluchajnyh funkcij $v$ determinirovannoj versii metoda Monte-Karlo $i$ zakon krasnogo smeshhenija [Optimum interpolation filtration of statistical characteristics for stochastic functions in determined version of Monte-Carlo method and the red shift law]. Moscow: Academy of sciences USSR.

19. Hinshaw G., Larson D., Komatsu E., Spergel D.N., Bennett C.L., Dunkley J., Nolta M.R., Halpern M., Hill R.S., Odegard N., Page L., Smith K.M., Weiland J.L., Gold B., Jarosik N., Kogut A., Limon M., Meyer S.S., Tucker G.S., Wollack E., Wright E.L. (2012). Nine-year Wilkinson microwave anisotropy probe observations: cosmological parameter results. Preprint WMAP. Draft Version. 
20. Spergel D.N., Verde L., Peiris H. V., Komatsu E., Nolta M. R., Bennett C. L., Halpern M., Hinshaw G., Jarosik N., Kogut A., Limon M., Meyer S. S., Page L., Tucker G. S., Weiland J. L., Wollack E., Wright E. L. (2003). First Year WMAP Observations: Determination of Cosmological Parameters. Astrophysical J. Suppl., Vol. 148, 175-194.

21. Larson D., Dunkley J., Hinshaw G., Komatsu E., Nolta M. R., Bennett C. L., Gold B., Halpern M., Hill R. S., Jarosik N., Kogut A., Limon M. (2011). Seven year WMAP observations: power spectra and WMAP-derived parameters. Astrophys. J. Suppl. Ser., № 192.

22. Arp H.C. (1992). Red shifts of high-luminosity stars - the K-effect, the Trumpler effect and mass-loss correction. Monthly Notices of the Royal Astronomical Society, Vol. 258, 800-810.

23. Wilkinson D.T., Partridge R.B. (1967). Large-scale density non-homogeneities in the Universe. Nature, 1967, vol. 215, № 5102.

24. Smoot G.F., Gorenstein M. V., Muller R. A. (1977). Detection of Anisotropy in the Cosmic Blackbody Radiation. Physical Review Letters, Vol. 39, № 14, 898-901.

25. Levin S.F. (2010). Izmeritel'naja zadacha identifikacii krasnogo smeshhenija [The Measurement problem of identification for red shift]. Metrology, № 5, 3-21.

26. Levin S.F. (2014). Cosmological distances scale. Part I. «Unexpected» Results. Measurement Techniques, Vol. 57, № 2, 117-122.

27. Vuchkov I., Boyadjieva L., Solakov E. (1987). Prikladnoj linejnyj regressionnyj analiz [The application linear regression analysis]. Moscow: Finance and statistics.

28. Förster E., Rönz B. (1979). Methoden der Korrelations- und Regressionsanalyse. Berlin: Verlag Die Wirtschaft.

29. Dunkley J., Komatsu E., Nolta M.R., Spergel D.N., Larson D., Hinshaw G., Page L., Bennett C.L., Gold B., Jarosik N., Weiland J.L., Halpern M., Hill R.S., Kogut A., Limon M., Meyer S.S., Tucker G.S., Wollack E., Wright E.L. (2009). 5-year WMAP observation: Likelihoods and Parameters from the WMAP data. Astrophys. J. Suppl., Vol. $180,306$.

30. Larson D., Dunkley J., Hinshaw G., Komatsu E., Nolta M.R., Bennett C.L., Gold B., Halpern M., Hill R.S., Jarosik N., Kogut A., Limon M., Meyer S. S., Odegard N., Page L., Smith K.M., Spergel D.N., Tucker G.S., Weiland J.L., Wollack E., Wright E. L. (2011). Seven year WMAP observations: power spectra and WMAP-derived pa-rameters. Astrophysical Journal Supplement Series, № 192, 16.

31. Levin S.F. (2011). Measurement problems in the statistical identification of the cosmological distances scale. Measurement Techniques, Vol. 54, № 12, 1334-1341. 
32. Freedman W.L., Madore B.F.,Gibson B.K., Ferrarese L., Kelson D.D., Sakai S., Mould J.R., Kennicutt R.C. Jr., Ford H.C., Graham J.A., Huchra J.P., Hughes S.M.G., Illingworth G.D., Macri L.M., Stetson P.B. (2001). Final Results from the Hubble Space Telescope Key Project to Measure the Hubble Constant. Astrophysical Journal, Vol. 553, 47-72.

33. Sazhin M.V. (2004). Anizotropija i poljarizacij reliktovogo izluchenij. Poslednie dannye [Anisotropy and polarisation of relic radiation. Last data]. Uspehi fizicheskih nauk [Advances in Physical Sciences], Vol. 174, № 2, pp. 197-205.

34. Hinshaw G. Hinshaw G., Larson D., Komatsu E., Spergel D.N., Bennett C.L., Dunkley J., Nolta M.R., Halpern M., Hill R.S., Odegard N., Page L., Smith K.M., Weiland J.L., Gold B., Jarosik N., Kogut A., Limon M., Meyer S.S., Tucker G.S., Wollack E., Wright E.L. (2012). Nine-year Wilkinson microwave anisotropy probe observations: cosmo-logical parameter results. Preprint WMAP. Draft Version.

35. Levin S.F. (2014), Cosmological distances scale. Part I. «Unexpected» Results. Measurement Techniques, Vol. 57, № 2, 117-122.

36. Planck Collaboration. (2014). Planck 2013 results. I. Overview of products and scientific results. Astronomy \& Astrophysics manuscript.

37. Planck Collaboration. (2014). Planck 2013 results. XVI. Cosmological parameters.

38. Riess A.G., Filippenko A.V., Challis P., Clocchiattia A., Diercks A., Garnavich P.M., Gilliland R.L., Hogan C.J., Jha S., Kirshner R.P., Leibundgut B., Phillips M.M., Reiss D., Schmidt B.P., Schommer R.A., Smith R.C., Spyromilio J., Stubbs C., Suntzeff N.B., Tonry J. (1998). Observational evidence from supernovae for an accelerating universe and a cosmological constant. Astronomical Journal, Vol. 116, 1009-1038.

39. Carroll S., Press W., Turner E. (1992). The Cosmological Constant. Annual Review of Astronomy \& Astrophysics, V.30, 499-542.

40. Riess A.G., Filippenko A.V., Challis P., Clocchiattia A., Diercks A., Garnavich P.M., Gilliland R.L., Hogan C.J., Jha S., Kirshner R.P., Leibundgut B., Phillips M.M., Reiss D., Schmidt B.P., Schommer R.A., Smith R.C., Spyromilio J., Stubbs C., Suntzeff N.B., Tonry J. (1998). Observational evidence from supernovae for an accelerating universe and a cosmological constant. Astronomical Journal, Vol. 116, 1009-1038.

41. Schwarz D.J., Weinhorst B. (2007). (An) isotropy of the Hubble diagram: comparing hemispheres. Astronomy \& Astrophysics, Vol. 474, 717-729.

42. Gosudarstvennaja sistema obespechenija edinstva izmerenij. Identifikacija raspredelenij verojatnostej pri reshenii izmeritel'nyh zadach [State system for ensuring the uniformity of 
Proceedings of International Conference PIRT-2015

measurements. Identification of probabilities distributions at the decision of measuring problems]. (2000).

43. Vinogradov I.M. (1979). Matematicheskaja jenciklopedija [Mathematical encyclopedia]. Vol. 2. Moscow: Soviet encyclopedia.

43. Schmidt B.P. (2011). The Path to Measuring an Accelerating Universe. Nobel Lecture. December 8, 2011. Nobel Foundation.

44. Tsvetkov D.Yu., Pavlyuk N.N., Bartunov O.S., Pskovskii Yu.P. (2005). Supernovae Catalogue. Moscow: State Astronomical Institute name of P.K. Sternberg. 